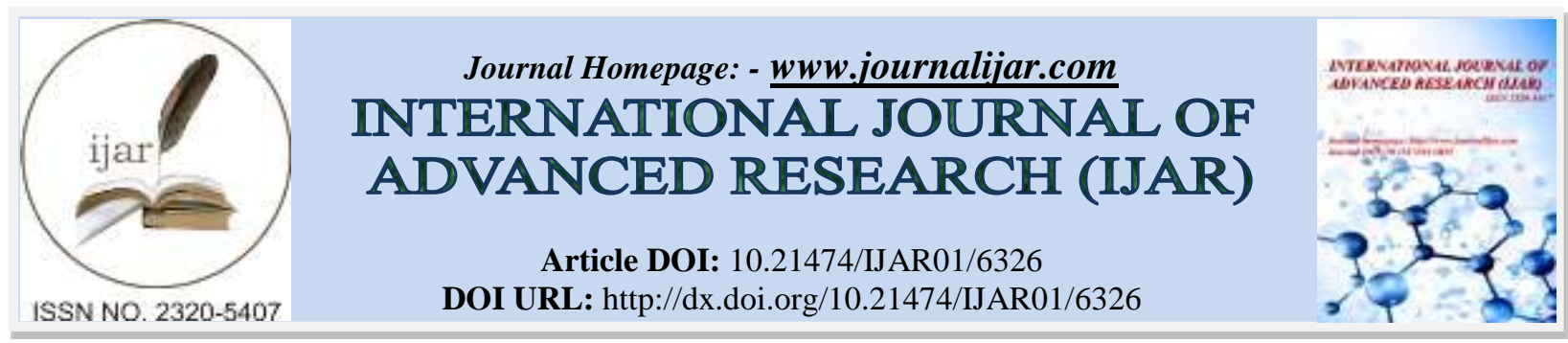

RESEARCH ARTICLE

\title{
EVALUATION OF THE IN VIVO ANTI-HELMINTHIC ACTIVITY, OF BRIDELIA MICRANTHA, CHENOPODIUM AMBROSOIDES AND OCIMUM AMERICANUM EXTRACTS AGAINST SCHISTOSOMA MANSONI INFECTION IN MICE.
}

"Tom Osebe ${ }^{1}$, Dorcas Yole ${ }^{2,3}$, David Odongo ${ }^{\mathbf{1}}$ and Horace Ochanda ${ }^{\mathbf{}}$.

1. School of Biological Sciences, University of Nairobi, P.O Box 30197-00100, Nairobi .

2. The Technical University of Kenya, P.O Box $52428-00200$, Nairobi.

3. Institute of Primate Research, P.O. Box 24481-00502, Karen, Nairobi.

\section{Manuscript Info}

(.........................

Manuscript History

Received: 17 November 2017

Final Accepted: 19 December 2017

Published: January 2018

Key words:-

Schistosomiasis, Praziquantel,

Histopathology, ELISA, Cytokine.

\section{Abstract}

Schistosomiasis is a chronic debilitating global disease affecting approximately 600 million people in 74 developing countries, with 800 million, mostly children at risk. Chemotherapy is the only immediate recourse to minimize the prevalence and incidence of this disease worldwide. Presently, Praziquantel is the only drug of choice for the treatment of all forms of schistosomiasis, however it shows low efficacy against schistosomula and juvenile stages. This dependence on a single drug with the likely potential for development of resistance to Praziquantel has justified the search for new alternative chemotherapies. Medicinal plants are potential candidates as sources of new drug prototypes. This study provides findings on the schistosomicidal activity of Bridelia micrantha, Chenopodium ambrosoides and Ocimum americanum plant extracts against Schistosoma mansoni infection in mice. Seven week old BALB/c mice were infected with approximately 250 cercariae and treated on the third and fourth week post infection with five crude extracts from the 3 plants for respective efficacy studies. Praziquantel treated group and infected control group served as controls. Perfusion was performed for all groups on the sixth week after infection for worm recovery. Worm recovery analysis confirmed that the three extracts have antischistosomal properties. Furthermore, pathology showed resolving granulomas and immune profiling results confirmed the extracts had immunomodulatory activity which could potentially be important in inhibiting infection.

Copy Right, IJAR, 2018,. All rights reserved.

\section{Introduction:-}

Human schistosomiasis which is caused by the trematode parasite Schistosoma, is included in the list of neglected diseases by the World Health Organization (WHO) and presents a significant economic as well as social impact. Chemotherapy is the only immediate recourse to minimize the prevalence and incidence of infection. Since human trials with Praziquantel were carried out in the late 1970s, the drug has gained prominence and today, it remains as the only treatment option available to 200 million people infected and 600 million at risk (Gonnert and Andrews 1977; Katz et al. 1979; Chitsulo et al. 2000; WHO, 2002; van der Werf et al. 2003; Fenwick et al. 2003; Cioli et al. 
2014). Although clinically insignificant, resistance has been reported in Egypt where some individuals could not be cured for schistosomiasis leading to identification of resistant isolates (Ismail et al. 1999). Studies have also shown that Praziquantel does not prevent re-infection and is not effective against schistosomula, which can result in low cure rates in areas where schistosomiasis is hyperendemic (Sabah et al. 1986). Praziquantel has further been reported to induce hemorrhage in the lung tissue of the host as well as abdominal pain and diarrhea (Flisser and McLaren 1989; Kabatereine et al. 2003). This has encouraged new studies in search of alternative therapies that could either replace or complement the use of Praziquantel in the treatment for schistosomiasis.

Natural products with therapeutic properties have been used for a long time. The main sources include mineral, plant and animal products (De Pasquale 1984). Plants have remained important sources contributing to $25 \%$ of prescribed drugs and $11 \%$ of drugs considered by WHO as essential (Rates 2001).

Bridelia micrantha, Ocimum americanum and Chenopodium ambrosoides have been used in traditional medicine to treat various ailments including as antihelminthics (Osebe et al. 2016). Their antischistosomal activity has also been reported in preliminary studies (Moilo et al. 2014; Waiganjo, Yole and Ochanda 2014). It will be interesting to determine if the extracts have immunomodulatory properties, a quality which has been observed for Praziquantel (Mutapi et al. 2005; Tweyongyere et al. 2011; Bourke et al. 2013). It will also be important to determine if the extracts have activity against 3 week old juvenile worms relative to Praziquantel.

\section{Materials and Methods:-}

\subsection{Plant material, extraction and preparation of extracts}

Bridelia micrantha (bark), Chenopodium ambrosoides (leaves and fruits) and Ocimum americanum (whole plant) were collected from various parts of Kenya. They were dried under shade at room temperature with periodic turning to prevent moulding until they were completely dry, then ground to fine powder with sieving through $0.5 \mathrm{~mm}$ mesh to standardize particle size.

To prepare water extracts, the ground powder was soaked in distilled water for $72 \mathrm{~h}$ then filtered through cotton wool and subsequently Whatman ${ }^{\circledR}$ qualitative filter paper, grade 1 (Sigma-Aldrich, Taufkirchen Germany). The filtrates were then frozen to $-10^{\circ} \mathrm{C}$ for $12 \mathrm{~h}$ and resultant ice blocks placed in vacuum chamber of a freeze dryer which was adjusted to $-100^{\circ} \mathrm{C}$ and 1 torr one at a time. Freeze drying was allowed to run until only a solid extract remained in the vacuum chamber.

To prepare hexane and methanol extracts $O$. americanum powder was soaked in analytical grade hexane (Unilabs, Nairobi Kenya) and C. ambrosoides fruit powder in analytical grade methanol (Pancreac Quimica, Barcelona Spain) for 72 h. Filtration was done first through cotton wool then using Whatman ${ }^{\circledR}$ qualitative filter paper grade 1 . The organic solvents were evaporated using a rotor evaporator (Joh, Achelis and Johne, Bremen Germany) at $70^{\circ} \mathrm{C}$ until pasty residues remained. The extracts were then transferred to small jars and further dried in fume chambers at room temperature for approximately 2 weeks.

\subsection{Infection of $B A L B / c$ mice with $S$. mansoni cercariae}

S. mansoni eggs recovered from chronically infected olive baboon (Papio anubis) was used to infect naïve snails (Biomphalaria pfeifferi) which were maintained at Institute of Primate Research's (IPR) malacology lab. The cercariae shed from snails were quantified and 250 used to infect each mouse. 7 week old BALB/c mice were used for this study where each group was composed of 10 mice being 5 of either sex. Infection was by ring method as described by Smithers and Terry (1965). The animals were maintained in cages each with 5 mice at IPR's rodent house with food pellets (Unga Group LTD, Kenya) and water ad libitum. Temperature was maintained at $20^{\circ} \mathrm{C} \pm$ $3^{\circ} \mathrm{C}$ and relative humidity at above $50 \%$ with natural day/night cycle.

\subsection{Treatment and worm recovery from infected mice}

The two mice treatment groups were treated by administering the preparation orally on the third and fourth week respectively and a dose being administered two days after the initial treatment. Dosage for the plant extracts treatment groups was $150 \mathrm{mg} / \mathrm{kg}$ as recommended in earlier studies (Moilo et al. 2014; Waiganjo, Yole and Ochanda, 2014). Praziquantel groups' dosage was $900 \mathrm{mg} / \mathrm{kg}$ and they served as drug control group. An infected control group was also included where treatment was withheld. The administered high dose of $900 \mathrm{mg} / \mathrm{kg} \mathrm{has} \mathrm{been}$ shown to be highly effective in mice while at the same time being within toxicity limits (Frohberg .1984; Muchirah et al. 2012). 
Worm recovery was performed on the sixth week post infection by perfusion using a perfusion pump (Manostat@, division of Barant Company, England) (Smithers and Terry 1965). Recovered worms from were manually counted and numbers analyzed as follows;

$$
\begin{aligned}
\text { Worm maturation } & =\frac{\text { Number of worms in } I C}{\text { Initial number of cercariae }} \times 100 \\
\text { Worm reduction } & =\frac{\text { Mean } I C-\text { Mean group }}{\text { Mean IC }} \times 100
\end{aligned}
$$

Test of significance was performed between the treatment groups using student's t-test for paired samples and ANOVA at $95 \%$ significance

\subsection{Gross pathology and histopathology analysis}

Fresh liver recovered from mice were observed for adhesion between liver lobes, general inflammation and presence of granuloma. The severity of granuloma presence per liver lobe was graded subjectively to None, Few (1-3), Moderate (4-10) and Severe (more than 10).

The livers were then fixed in $10 \%$ formaldehyde, processed as per routine and sectioned to $0.7 \mu \mathrm{m}$ thickness using a microtome before mounting on slides (Hopwood 1996; Carson 2007; Carson and Christa 2009). Staining was done using haematoxylin and eosin dyes. Under an ocular micrometer, 10 granulomas from each animal's liver were measured on their vertical and horizontal dimensions, the average of which was assumed to be the granuloma diameter (Farah et al. 2000).

\subsection{Immune reaction of treatment groups}

\subsubsection{ELISA and Flow cytometry reagents and kits}

ELISA 96 well plates (Nunc-Immuno ${ }^{\mathrm{TM}}$ plate marxi sorp ${ }^{\mathrm{TM}}$ ) and goat anti-mouse IgG were obtained from SigmaAldrich, USA while the substrate (SureBlue ${ }^{\mathrm{TM}}$, TMB microwell peroxidase substrate -1 component) was procured from KPL, Gaithersburg USA.

All materials used in flow cytometry were obtained from BD Biosciences (Califonia, USA). BD cytometric bead array (CBA) mouse Th1/Th2 cytokine kit contained all the necessary reagents and capture antibodies specific for IL-

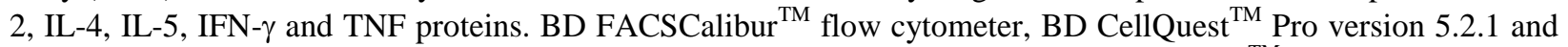
FCap Array software version 1 were used in the analysis of cytokine profiles. BD Falcon ${ }^{\mathrm{TM}} 12 \times 75 \mathrm{~mm}$ sample acquisition tubes for the flow cytometer and $15 \mathrm{ml}$ conical propylene tubes were also used.

Serum was prepared from blood that was recovered from mice during perfusion. The blood was initially left on the bench to clot at room temperature then kept at $4^{\circ} \mathrm{C}$ overnight. The following morning, the blood was retrieved and centrifuged at $450 \mathrm{~g}$ at $4^{\circ} \mathrm{C}$ for 15 minutes then supernatant recovered and stored in $1 \mathrm{ml}$ microfuge tubes at $-20^{\circ} \mathrm{C}$ for subsequent studies.

\subsubsection{Determination of specific IgG profiles of the different treatments using ELISA}

Soluble egg antigen (SEA), 0-3 h release protein (0-3 h) and soluble adult worm antigen preparation (SWAP) antigens were diluted in $1 \mathrm{X}$ PBS at a concentration of $1 \mathrm{mg} / \mathrm{ml}$ and $50 \mu \mathrm{l}$ of each added to ELISA plates wells. This was allowed to stand overnight at $4^{\circ} \mathrm{C}$ then washed three times using wash buffer with ELISA washer system (Dynex Technologies LTD, Guernsey channel Great Britain).

After draining all wash buffer, $100 \mu \mathrm{l}$ blocking buffer was added to each well and allowed to stand at $37^{\circ} \mathrm{C}$ for $1 \mathrm{~h}$, then washed three times. $50 \mu \mathrm{l}$ of antibody mixture prepared by mixing mice serum with blocking buffer at a concentration of $2.5 \mu \mathrm{l} / \mathrm{ml}$ was added to each well in duplicate and allowed to stand at $37^{\circ} \mathrm{C}$ for $2 \mathrm{~h}$. This was washed six times and $50 \mu \mathrm{l}$ conjugate goat anti-mouse $\mathrm{IgG}$ in a dilution of $1 \mu \mathrm{l} / 2 \mathrm{ml}$ of $1 \% \mathrm{BSA}$ added then incubated at $37^{\circ} \mathrm{C}$ for $1.5 \mathrm{~h}$. The plates were then washed six times and $50 \mu \mathrm{l}$ of substrate added. This was allowed to stand in a dark room for $30 \mathrm{~min}$ and reading done using ELISA reader (Dynatech Laboratories, USA) at OD 450. Test of significance of the mean OD readings was performed using ANOVA at $95 \%$ significance

\subsubsection{Determination of cytokine profiles of the different treatments using flow cytometry}

1.5.3.1 Mixing of mouse Th1/Th2 cytokine capture beads and cytokine assay

Reconstitution and serial dilution of standards was done according to manufacturer instructions. Mixing of mouse Th1/Th2 cytokine capture beads was also done as per manufacturer instructions. Briefly, each of the Capture Bead suspension was individually vortexed and $10 \mu \mathrm{l}$ aliquot of each pooled in a single tube labeled "Mixed Capture 
Beads" and vortexed thoroughly. $50 \mu 1$ of the Th1/Th2 cytokine standard solutions were added to control tubes as indicated by manufacturer.

The test groups' serum samples were retrieved and $50 \mu 1$ of each added to an acquisition tube then $50 \mu 1$ of mouse Th1/Th2 PE Detection Reagent added. This mixture was then incubated in darkness for $2 \mathrm{~h}$ at room temperature. $1 \mathrm{ml}$ of wash buffer was added to each of the tubes and centrifuged at $200 \mathrm{~g}$ for $5 \mathrm{~min}$ then supernatant discarded. $300 \mu 1$ of wash buffer was then added to resuspend the bead pellet and suspension loaded on BD FACSCalibur ${ }^{\mathrm{TM}}$ flow cytometer. The data was acquired using BD CellQuest ${ }^{\mathrm{TM}}$ Pro version 5.2.1 and analyzed using FCap Array software version 1. Test of significance of mean cytokine concentrations was performed using ANOVA at $95 \%$ significance.

\section{Results:-}

Worm efficacy results are summarized in Tables 1 and 2 below.

1.6 Effect of the plant extracts on the survival of 4 week and 3 week old schistosomes

Other than $O$. americanum water extract, all treatments were significantly better than IC for the 4 week old worms efficacy study (Table 1). For the 3 week old worms efficacy study, all treatments were significantly better than IC except for $B$. micrantha water extract (Table 2). Praziquantel was highly effective against both three and four weeks old worms

\subsection{Four and week old worms treatment groups liver gross pathology analysis}

Results of liver gross pathology are shown in Tables 3 and 4.

Adhesion between liver lobes was observed for all treatment groups. All the treatment groups in the 4 week old worms efficacy study except $B$. micrantha water extract and $O$. americanum hexane extract presented with few to no observable granulomas correlating with general inflammation observations. $C$. ambrosoides water extract, $O$. americanum water extract and Praziquantel treatment groups had no observable general inflammation.

All the treatment groups for the 3 week old worms efficacy study except $C$. ambrosoides water extract, $O$. americanum hexane extract and $O$. americanum water extract presented with moderate, few to no observable granulomas. B. micrantha water extract and Praziquantel had no observable inflammation which correlates with the few to no observable granulomas observed

\subsection{Histopathology of mice liver biopsies}

Using one way ANOVA $(\dot{\alpha}=0.05)$ the means were significantly different although the post hoc Tukey HSD did not detect any differences between paired means. In decreasing order, the average granuloma sizes were infected control $(240.9 \mu \mathrm{m})$, O. americanum water extract $(234.6 \mu \mathrm{m})$, C. ambrosoides methanol extract $(171.1 \mu \mathrm{m})$, O. americanum hexane extract $(170 \mu \mathrm{m})$, Praziquantel $(160 \mu \mathrm{m}), B$. micrantha water extract $(153.2 \mu \mathrm{m})$ and finally $C$. ambrosoides water extract $(70 \mu \mathrm{m})($ Figure 1$)$.

Histopathology changes -determined mainly by extent of lymphocytic cellular infiltration- of liver from group treated with Praziquantel was mild with few areas of necrosis and granuloma. Other than for B. micrantha group where histopathology changes were moderate, the other groups varied ranging from mild to severe. Infected control group had highest number of granuloma per slide and none was observed to be resolving. The other treatment groups however had granuloma which were resolving being without a distinct central egg. The photomicrographs of the liver sections are shown in Figure 2.

\subsection{IgG profiles of the different treatment groups using ELISA}

There was no statistical difference for IgG profiles using SWAP antigen in 4 week old worm treatment study. OD readings in decreasing order were $C$. ambrosoides methanol extract treatment $(0.586), B$. micrantha water extract (0.537), C. amrosoides water extract (0.517), Praziquantel (0.510), infected control (0.448), naïve (0.438), $O$. americanum water extract $(0.433)$ and $O$. americanum hexane extract $(0.397)$. The mean OD readings in 3 week old worms treatment study were generally lower when compared to the 4 week old worms except for $B$. micrantha water extract (0.619). Mean OD readings for the other treatment groups in decreasing order were $C$. ambrosoides methanol extract (0.515), $C$. ambrosoides water extract (0.494), O. americanum water extract (0.431), Praziquantel (0.390) and $O$. americanum hexane extract (0.331). Same naïve OD readings applies for 3 week worm efficacy study. AntiSWAP IgG profile mean OD readings are graphically represented in figures 3 and 4. 
IgG profile against the schistosomular 0-3 hour release protein had mean OD readings that were lower than both SWAP and SEA. There was no statistical difference in 4 week or 3 week worm treatment groups. The readings in decreasing order for 4 week worm treatment groups were B. micrantha water extract $(0.266)$, C. ambrosoides methanol extract and praziquantel at $0.226, O$. americanum hexane (0.214), infected control $0.210, C$. ambrosoides water extract (0.202), O. americanum water extract (0.194) and naïve (0.180). In 3 week old worm treatment study, the mean OD readings in decreasing order were B. micrantha water extract (0.257), C. ambrosoides methanol extract $0.202, C$. ambrosoides water extract and $O$. americanum hexane extract $(0.185), O$. americanum water extract (0.172) and Praziquantel (0.170). Same naïve OD readings applies for 3 week old worms efficacy study. Anti- 0-3 hour release protein profile is graphically represented in figures 3 and 4.

Anti-SEA IgG profile mean OD readings were also not significantly different for 4 week or 3 week old worm treatment groups. Naïve OD reading was however statistically lower when compared with the 4 week worm treatment groups. Decreasing order of mean OD readings in 4 week worm treatment was $C$. ambrosoides methanol extract (0.519), B. micrantha water extract (0.461), C. ambrosoides water extract (0.427), Praziquantel (0.428), $O$. americanum water extract (0.353), infected control $(0.330)$, O. americanum hexane extract $(0.301)$ and naïve (0.284). The decreasing order in 3 week old worms treatment experiment was B. micrantha water extract (0.473), $C$. ambrosoides water extract (0.437), C. ambrosoides methanol extract (0.411), O. americanum water extract (0.381), Praziquantel (0.367), O. americanum hexane extract (0.323). Same naïve OD readings applies for 3 week old worms efficacy study. Anti-SEA IgG profile is graphically represented in figures 3 and 4 below.

\subsection{Cytokine profiles of the different treatment groups using Flow cytometry}

Statistical difference for 4 week old efficacy study was observed for all cytokine concentration groups except for IL4 which was only detectable for mice treated with $B$. micrantha water extract. Infected control and naïve cytokine concentrations were lower than in all treatment groups except for IL-2 where $C$. ambrosoides water extract and $B$. micrantha water extract treatment groups had lower concentrations. The cytokine concentrations for treatment groups is summarized in table 5 .

Statistical difference for 3 week old worm efficacy study was observed for TNF and IL-5. IL4 was only detectable for mice treated with $C$. ambrosoides methanol extract. Infected control had IL-2 concentration of $4.91 \mathrm{pg} / \mathrm{ml}$ which was higher than for four treatment groups including Praziquantel. Naïve IL-2 concentration was lower than for all treatment groups except $B$. micrantha water extract. These differences were however not statistically significant. IFN- $\gamma$ also lacked a statistical difference but concentration for infected control was relatively low and undetectable for naïve group. The cytokine concentrations for treatment groups is summarized in table 6 .

\section{Discussion:-}

In recent years, a substantial interest in natural products to the treatment of Neglected Tropical Diseases, including schistosomiasis, has been growing. The interest has been exploited and stimulated as an effort to develop a new medicine as an alternative method to this parasitosis treatment. This study investigated the in vivo efficacy of Bridelia micrantha, Ocimum americanum and Chenopodium ambrosoides against 3 and 4 week old worms of $S$. mansoni.

Generally, all treatments were more effective than the untreated control group suggesting they contain phytochemicals that have activity against S. mansoni. Treatment with Praziquantel - which acted as drug control group- was as expected, observed to be more effective than the crude plant extracts. Crude plant extracts typically contain many different biomolecules and their concentration may be suboptimal relative to the pure active ingredient in Praziquantel.

It was interesting to observe that $O$. americanum water extract was more effective against 3 week old worms while B. micrantha water extract was more effective against 4 week old worms. By week three after infection, majority of the worms are already in the portal blood stream (Doenhoff et al. 1978), where they remain in adult stage. This means the worms were mostly exposed to the treatment in the same location. Although it is not known which antigens, it is suggested that tegumental antigens of schistosomes vary as the worms mature from cercaria to schistosomule to adult which is thought to be a protective mechanism (Sepulveda et al. 2010). This therefore suggests there are worm structural differences that may have led to the two extracts having more pronounced activity in either the 3 week or 4 week old worms. 
Our findings were however not consistent with studies performed by Sabah et al (1986), since Praziquantel was observed to be as effective against the juvenile worms. This may be due to differences in experimental design where we exposed the mice to $900 \mathrm{mg} / \mathrm{kg}$ dose compared to $250 \mathrm{mg} / \mathrm{kg}$ in Sabah's study. There may also be some differences between the Kenyan strain of $S$. mansoni when compared to the Puerto Rican one.

While the pathology for the plant extracts was not as remarkable as for the Praziquantel treated groups, the observation of resolving granulomas could be an indicator that the extracts have comparable properties noting that the eggs were produced by adult female worms in the days following treatment for both studies. Potentially at higher doses than the administered $150 \mathrm{mg} / \mathrm{kg}$, the effect would be more pronounced. Alternatively, pathology of mice done serially over a few weeks would better show the disease progression. IgG responses were observed to be similar across the treatment groups, infected control and naïve despite variation in worm numbers suggesting insignificant IgG effect in early stage infection. Naïve OD readings were potentially due to non specific binding

Interestingly, Praziquantel profile was not highest for any of the cytokines while infected control was low in all groups except for IL-2. While it is known that innate immunity can develop following prolonged exposure owing to responses against dead worms (Mitchell et al. 2012), Praziquantel has been observed to elicit protective immune responses (Mutapi et al. 2005; Bourke et al. 2012).

The balance of Th1 and Th2 responses is important in the pathology of schistosomiasis. Th1 responses are associated with the acute stage of the infection being a reaction towards the worms while Th2 responses occur later due to eggs laid by mature worms (Cintron-Rivera 1956; Warren 1973; Nash et al. 1982; McManus and Loukas, 2008). Th2 associated cytokines IL-4, IL-5 and IL-13 all play important roles in pathogenesis of schistosomiasis with IL-4 and IL-13 directing granuloma formation (Chiaramonte et al. 1999a). IL-13 on the other hand is primarily the stimulus for tissue fibrosis (Chiaramonte et al. 1999b; Fallon et al. 2000). Prominence of Th1 cytokines especially IFN $\gamma$ has an effect of down regulating Th2 cytokines and consequently a reduction of granuloma size and fibrosis (Cheever. 1992; Cheever et al. 1998). IL-5 serves to recruit eosinophils but has no obvious effect on granuloma size, IL-4 leads to formation of IgE with varied effect on granuloma while IL-2 increases granuloma size and fibrosis (Cheever. 1992). Although TNF has not been widely studied, it is thought to have a role in periportal fibrosis (Cheever. 1992; Ramadan et al. 2013). This suggests the inflammation causing cytokines may be important in acting against the miracidia within eggs and thereafter, other cytokines resolve the granuloma implying the importance of the cytokine balance. This suggests the plant extracts elicited cytokine responses that compared well with Praziquantel and may potentially be protective.

\section{Conclusion:-}

According to the results the plant extracts used in this study could be developed to be important alternatives to Praziquantel offering antischistosomal activity, improve pathology upon treatment and elicit mechanisms for protection to reinfection. Therefore, this study opens up perspectives for future researches on the substance or the compound isolation, making possible potential new treatment alternatives against $S$. mansoni. While the toxicology of these plants extracts have been studied where B. micrantha water extract was determined to be relatively toxic, the drug candidates will also need to be assessed in terms of metabolism, chemistry and treatment regimen (Nwaka and Riley 2003; Osebe et al. 2016)

\section{Aknowledgements:-}

This work was financially supported by National Commission for Science, Technology and Innovation (third call) and Deutsche Akademischer Austauschdienst (DAAD). The authors are also grateful to Stanley Mathenge, Nicodemus Muia, Collins Ngudi, Sammy Kisara and Simon Kiarie for technical support in this study 


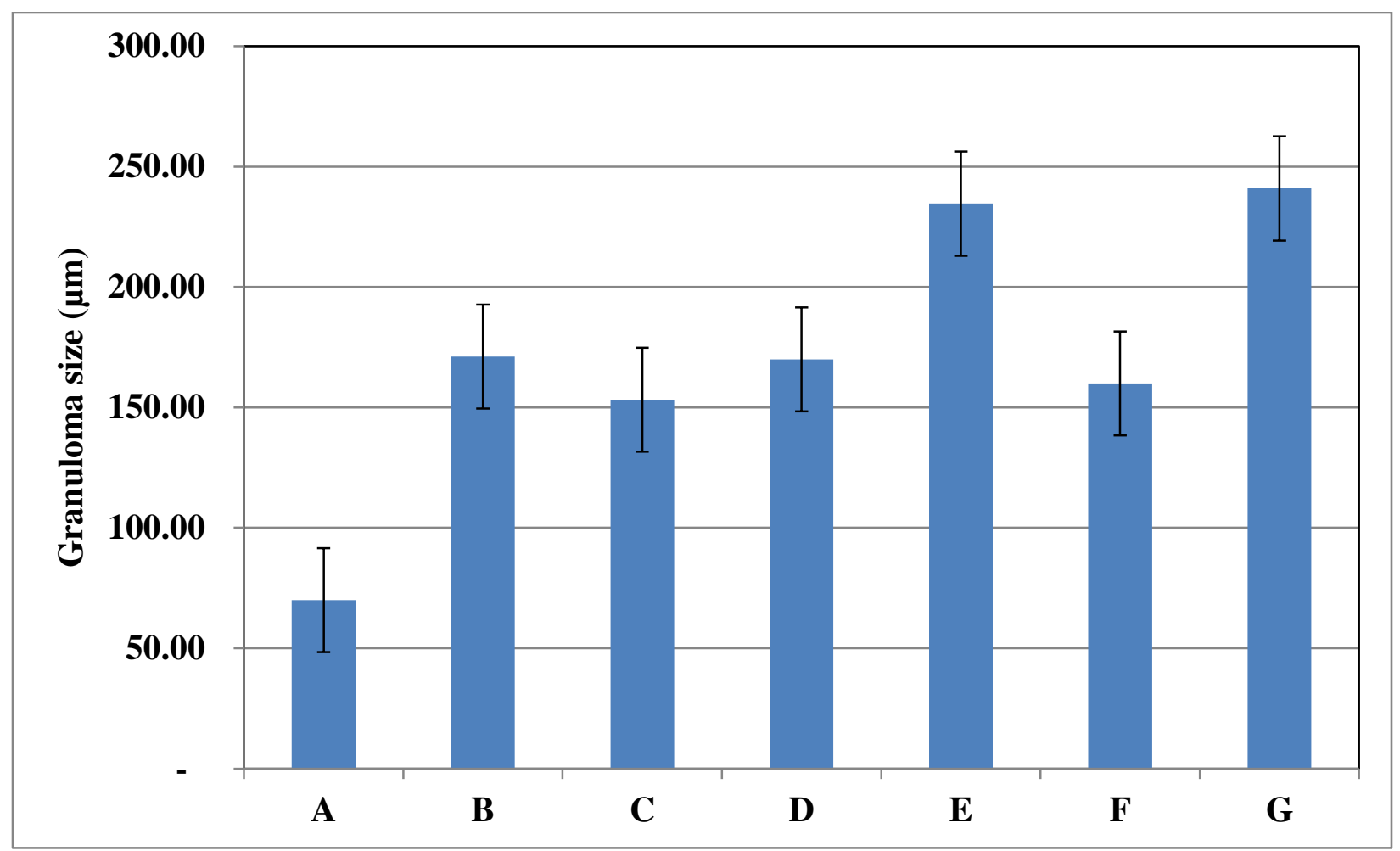

Figure 1:- Mean hepatic granuloma diameter of BALB/c mice groups treated with plant extracts, Praziquantel and infected control where treatment was withheld. Statistical difference was tested using ANOVA $(\dot{\alpha}=0.05)$ and post hoc Tukey HSD to determine the groups that differ significantly

Key: A (C. ambrosoides water extract); B (C. ambrosoides methanol extract); C (B. micrantha water extract); D (O. americanum hexane extract); E (O. americanum hexane extract); F (Praziquantel); G (Infected control).
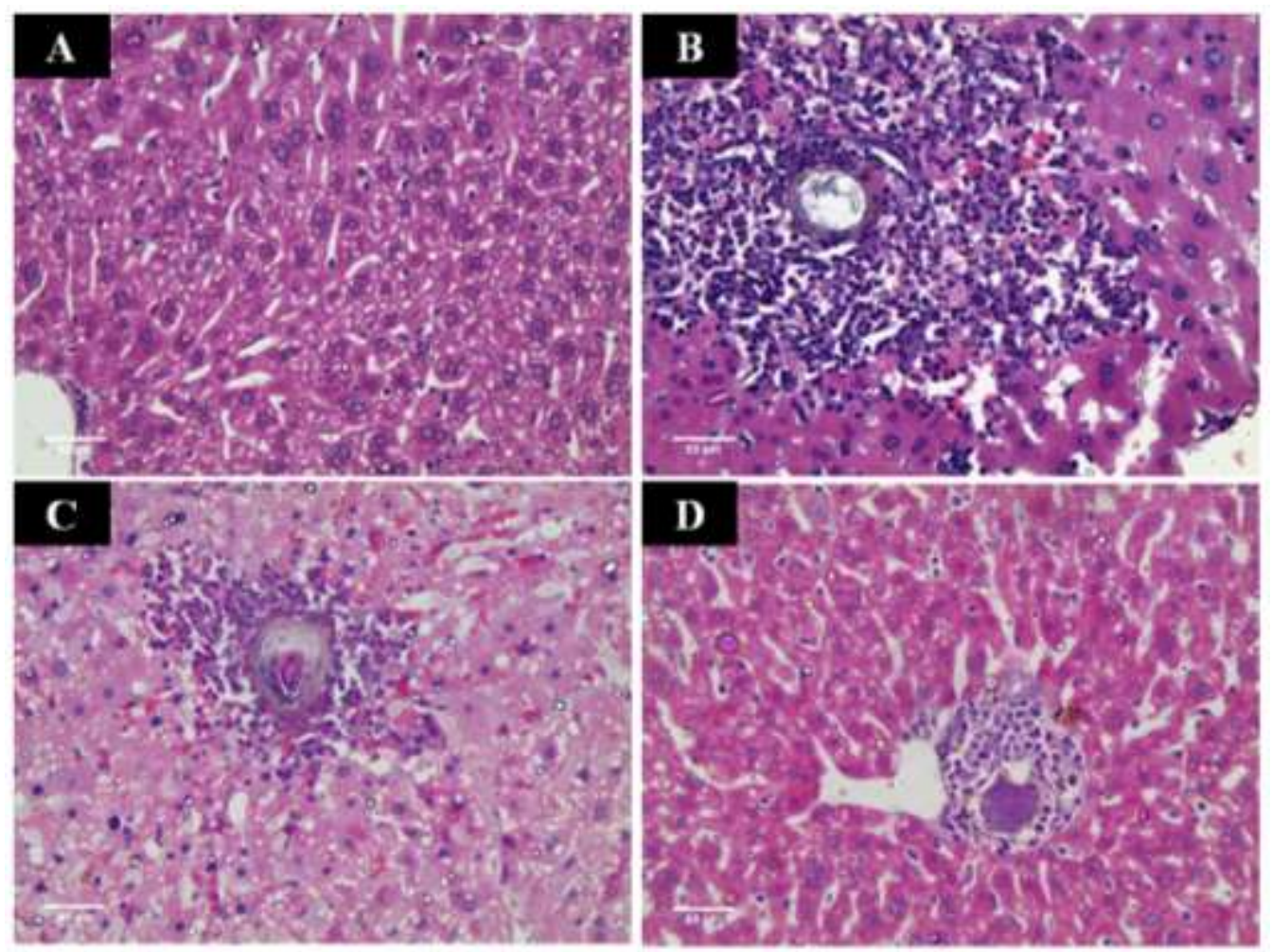

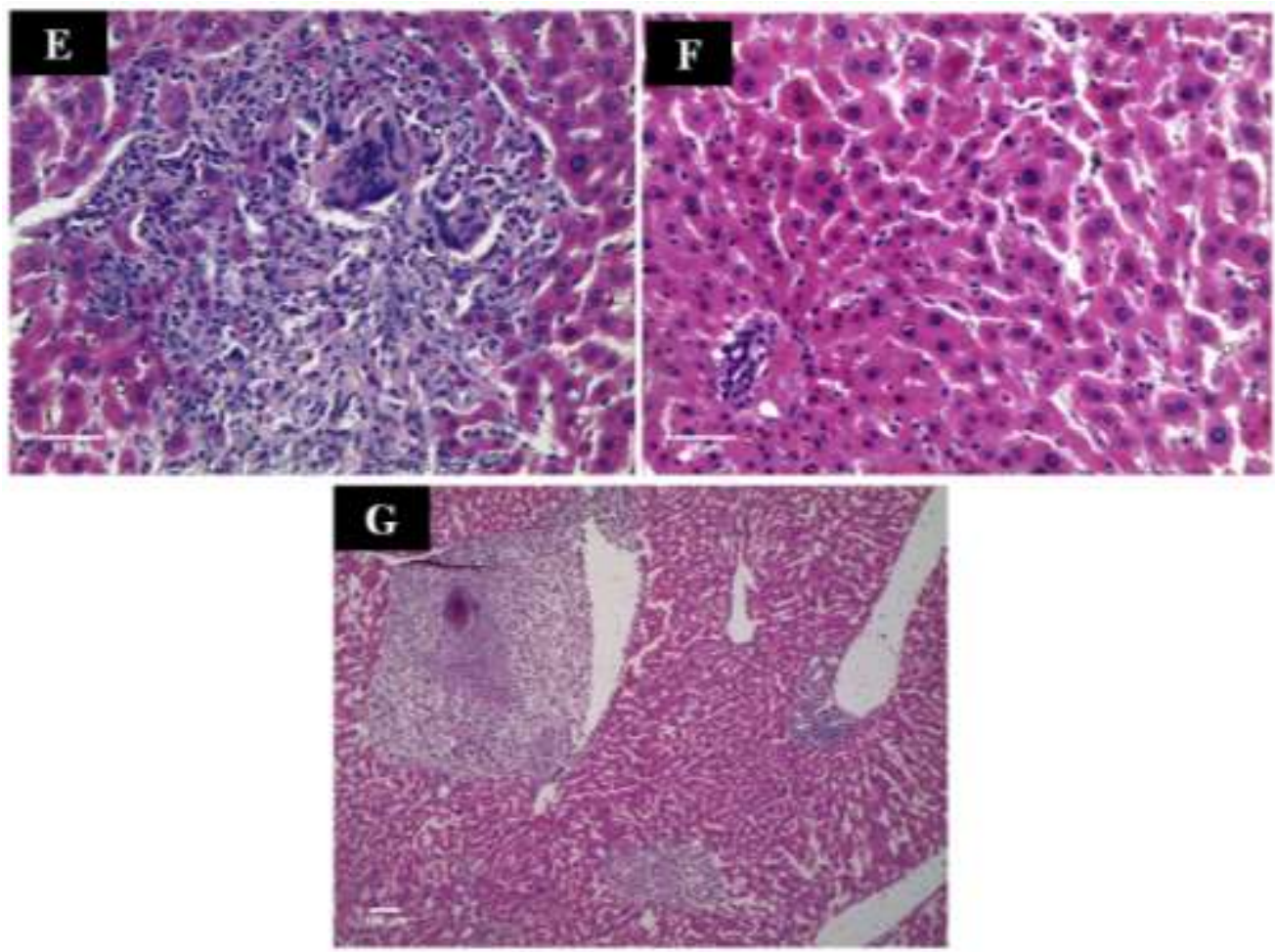

Figure 2:- Histological sections of livers of BALB/c mice groups treated with plant extracts, praziquantel and infected control where treatment was withheld. Granuloma with intact egg was observed for the infected control and resolving granulomas for the treatment groups. Note that histological section for infected control was taken at a lower magnification than the others in order to visualize the entire granuloma

Key: A (Praziquantel); B (B. micrantha water extract); C (C. ambrosoides methanol extract); D (C. ambrosoides water extract); E (O. americanum hexane extract); F (O. americanum water extract); G (Infected control).

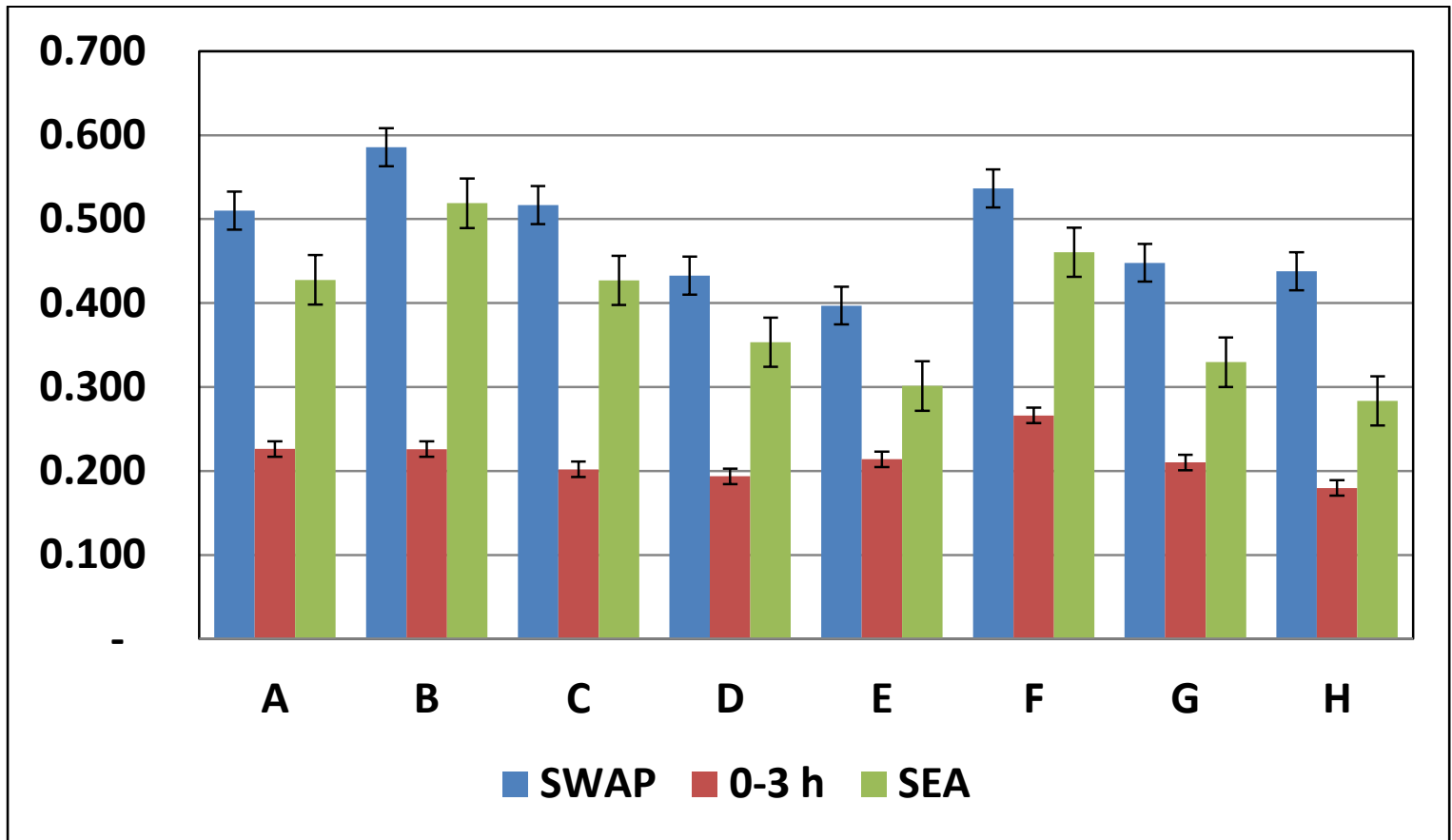

Figure 3:- IgG mean OD readings of mice infected with S. mansoni and treated on week 4 post infection. Statistical difference was tested using ANOVA $(\dot{\alpha}=0.05)$ 
Key: A - Praziquantel; B - C. ambrosoides methanol extract; C - C. ambrosoides water extract; D - O. americanum water extract; E - O. americanum hexane extract; F - B. micrantha water extract; $\mathrm{G}$ - infected control; $\mathrm{H}$ - non infected control

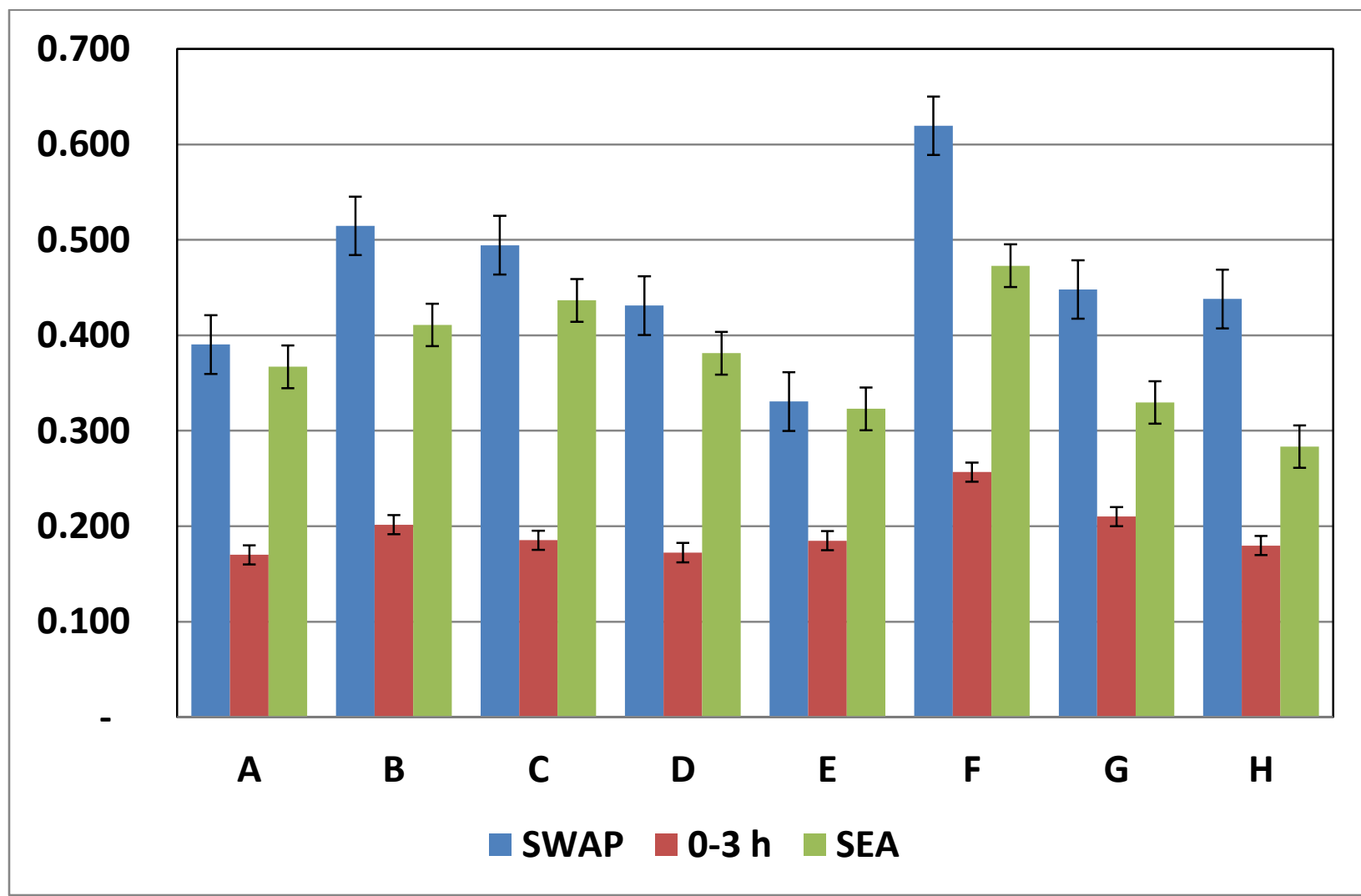

Figure 4:- IgG mean OD readings of mice infected with S. mansoni and treated on week 3 post infection. Statistical difference was tested using ANOVA ( $\dot{\alpha}=0.05)$

Key: A - Praziquantel; B - C. ambrosoides methanol extract; C - C. ambrosoides water extract; D - O. americanum water extract; E - O. americanum hexane extract; F - B. micrantha water extract; G - infected control; H - non infected control

Table 1:- Results of 4 week old S. mansoni treatment with selected plant extracts relative to standard treatment. The mice were infected with estimated 250 cercariae and treated on week 4 post infection. Statistical difference was analyzed using ANOVA $(\dot{\alpha}=0.05)$ and paired difference using students t-test $(\mathrm{p}>0.05)$

\begin{tabular}{|l|l|l|l|l|}
\hline & MEAN \pm SE & Total & \\
\hline Treatment & Males & Females & \% Worm Reduction \\
\hline C. ambrosoides(water) & $30.5 \pm 3.5$ & $16 \pm 4$ & $46.5 \pm 0.5$ & $33 \%$ \\
\hline C. ambrosoides(methanol) & $29.29 \pm 3.34$ & $16.29 \pm 2.01$ & $45.57 \pm 4.93$ & $35 \%$ \\
\hline B. micrantha(water) & $28.25 \pm 4.14$ & $16.13 \pm 2.75$ & $44.38 \pm 6.62$ & $36 \%$ \\
\hline O. americanum(hexane) & $30.33 \pm 3.91$ & $17.5 \pm 3.4$ & $47.83 \pm 6.79$ & $31 \%$ \\
\hline O. americanum(water) & $38.14 \pm 3.89$ & $19.57 \pm 3.16$ & $57.71 \pm 6.91$ & $17 \%$ \\
\hline Praziquantel & $10.11 \pm 1.49$ & $3.00 \pm 0.53$ & $13.11 \pm 1.39$ & $81 \%$ \\
\hline Infected Control & $46.67 \pm 2.96$ & $23 \pm 2.51$ & $69.67 \pm 4.98$ & \\
\hline
\end{tabular}

Table 2:- Results of 3 week old S. mansoni treatment with selected plant extracts relative to standard treatment. The mice were infected with estimated 250 cercariae and treated on week 3 post infection. Statistical difference was analyzed using ANOVA $(\dot{\alpha}=0.05)$ and paired difference using students t-test $(\mathrm{p}>0.05)$

\begin{tabular}{|l|l|l|l|l|}
\hline \multicolumn{3}{|l|}{ MEAN \pm SE } & \\
\hline Treatment & Males & Females & Total & \% Worm Reduction \\
\hline C. ambrosoides (water) & $31.88 \pm 3.44$ & $15 \pm 3.22$ & $46.88 \pm 6.12$ & $33 \%$ \\
\hline
\end{tabular}




\begin{tabular}{|l|l|l|l|l|}
\hline C. ambrosoides (methanol) & $28.75 \pm 3.09$ & $15.25 \pm 2.95$ & $44 \pm 5.94$ & $37 \%$ \\
\hline B. micrantha (water) & $41.57 \pm 4.89$ & $20.71 \pm 2.43$ & $62.29 \pm 7$ & $11 \%$ \\
\hline O. americanum (hexane) & $27.4 \pm 6.65$ & $14.8 \pm 4.72$ & $42.2 \pm 10.82$ & $39 \%$ \\
\hline O. americanum (water) & $25.83 \pm 4.9$ & $13.17 \pm 3.63$ & $39 \pm 8.49$ & $44 \%$ \\
\hline Praziquantel & $11 \pm 3.39$ & $5 \pm 1.41$ & $16 \pm 4.49$ & $77 \%$ \\
\hline Infected Control & $46.67 \pm 2.96$ & $23 \pm 2.51$ & $69.67 \pm 4.98$ & \\
\hline
\end{tabular}

Table 3:- Liver gross pathology analysis in 3 week old worm treatment groups. The mice were infected with estimated 250 cercariae and treated on week 3 post infection followed by perfusion on week 6 .

\begin{tabular}{|l|l|l|l|l|l|l|l|}
\hline & & Adhesion & Inflamation & \multicolumn{3}{|c|}{ Granuloma categorization } \\
\hline Treatment & No. of mice & Yes & No & Severe & Moderate & Few & None \\
\hline C. ambrosoides (water extract) & 8 & $100 \%$ & $100 \%$ & $0 \%$ & $0 \%$ & $50 \%$ & $50 \%$ \\
\hline C. ambrosoides (methanol extract) & 7 & $100 \%$ & $86 \%$ & $0 \%$ & $0 \%$ & $43 \%$ & $57 \%$ \\
\hline B. micrantha (water extract) & 7 & $100 \%$ & $57 \%$ & $0 \%$ & $14 \%$ & $71 \%$ & $14 \%$ \\
\hline O. americanum (hexane extract) & 6 & $100 \%$ & $67 \%$ & $0 \%$ & $17 \%$ & $50 \%$ & $33 \%$ \\
\hline O. americanum (water extract) & 6 & $100 \%$ & $100 \%$ & $0 \%$ & $0 \%$ & $33 \%$ & $67 \%$ \\
\hline Praziquantel & 4 & $100 \%$ & $100 \%$ & $0 \%$ & $0 \%$ & $25 \%$ & $75 \%$ \\
\hline Infected Control & 6 & $100 \%$ & $17 \%$ & $0 \%$ & $67 \%$ & $33 \%$ & $0 \%$ \\
\hline
\end{tabular}

\section{Granuloma classification Key:}

None -No granuloma observed per lobe; Few- 1 to 3 granuloma per lobe; Moderate- 4 to 10 granuloma per lobe;

Severe- more than 10 granuloma per lobe

Table 4:- Liver gross pathology analysis in 4 week old worm treatment groups. The mice were infected with estimated 250 cercariae and treated on week 4 post infection followed by perfusion on week 6

\begin{tabular}{|c|c|c|c|c|c|c|c|}
\hline & & Adhesion & Inflamation & \multicolumn{4}{|c|}{ Granuloma categorization } \\
\hline Treatment & No. of mice & Yes & No & Severe & Moderate & Few & None \\
\hline C. ambrosoides (water extract) & 7 & $100 \%$ & $86 \%$ & $14 \%$ & $0 \%$ & $29 \%$ & $57 \%$ \\
\hline C. ambrosoides (methanol extract) & 7 & $100 \%$ & $86 \%$ & $0 \%$ & $14 \%$ & $57 \%$ & $29 \%$ \\
\hline B. micrantha (water extract) & 8 & $100 \%$ & $100 \%$ & $0 \%$ & $0 \%$ & $13 \%$ & $88 \%$ \\
\hline O. americanum (hexane extract) & 7 & $100 \%$ & $57 \%$ & $14 \%$ & $29 \%$ & $14 \%$ & $43 \%$ \\
\hline O. americanum (water extract) & 7 & $100 \%$ & $43 \%$ & $17 \%$ & $50 \%$ & $17 \%$ & $17 \%$ \\
\hline Praziquantel & 9 & $100 \%$ & $100 \%$ & $0 \%$ & $0 \%$ & $89 \%$ & $11 \%$ \\
\hline Infected Control & 6 & $100 \%$ & $17 \%$ & $0 \%$ & $67 \%$ & $33 \%$ & $0 \%$ \\
\hline
\end{tabular}

\section{Granuloma classification Key:}

None -No granuloma observed per lobe; Few- 1 to 3 granuloma per lobe; Moderate- 4 to 10 granuloma per lobe; Severe- more than 10 granuloma per lobe

Table 5:- Mean cytokine concentrations of mice treated against 4 week old worms. Serum was prepared from blood drawn from BALB/c mice treatment groups then analyzed using flow cytometry. Statistical difference was analyzed using ANOVA $(\dot{\alpha}=0.05)$ and paired difference using students t-test $(\mathrm{p}>0.05)$

\begin{tabular}{|c|c|c|c|c|c|}
\hline \multirow[b]{3}{*}{ Treatment groups } & \multicolumn{5}{|c|}{ Cytokine concentration in pg/ml } \\
\hline & \multicolumn{3}{|c|}{ T-helper 1} & \multicolumn{2}{|c|}{ T-helper 2} \\
\hline & TNF & IFN- $\gamma$ & IL-2 & IL-5 & IL-4 \\
\hline Praziquantel & 12.68 & 2.88 & 6.13 & 5.63 & - \\
\hline C. ambrosoides (water extract) & 6.67 & 3.73 & 1.96 & 12.28 & - \\
\hline C. ambrosoides (methanol extract) & 105.03 & 37.12 & 7.78 & 8.31 & - \\
\hline B. micrantha (water extract) & 12.51 & 2.49 & 2.02 & 29.80 & 11.56 \\
\hline O. americanum (hexane extract) & 9.27 & - & 14.6 & 11.74 & - \\
\hline O. americanum (water extract) & 5.66 & 4.07 & - & 13.74 & - \\
\hline Infected control & 3.53 & 0.26 & 4.91 & - & - \\
\hline Naïve & 3.81 & - & 2.72 & 2.46 & - \\
\hline
\end{tabular}


Table 6:- Mean cytokine concentrations of mice treated against 3 week old worms. Serum was prepared from blood drawn from BALB/c mice treatment groups then analyzed using flow cytometry. Statistical difference was analyzed using ANOVA $(\alpha=0.05)$ and paired difference using students t-test ( $p>0.05)$

\begin{tabular}{|c|c|c|c|c|c|}
\hline \multirow[b]{3}{*}{ Treatment groups } & \multicolumn{5}{|c|}{ Cytokine concentration in pg/ml } \\
\hline & \multicolumn{3}{|c|}{ T-helper 1} & \multicolumn{2}{|c|}{ T-helper 2} \\
\hline & TNF & IFN- $\gamma$ & IL-2 & IL-5 & IL-4 \\
\hline Praziquantel & 8.54 & 1.20 & 3.66 & 4.76 & - \\
\hline C. ambrosoides (water extract) & 58.68 & 1.32 & 5.78 & 8.48 & - \\
\hline C. ambrosoides (methanol extract) & 6.30 & 0.86 & 6.92 & 9.14 & 6.16 \\
\hline B. micrantha (water extract) & 11.54 & 1.70 & 2.54 & 17.86 & - \\
\hline O. americanum (hexane extract) & 9.59 & 0.99 & 4.59 & 9.45 & - \\
\hline O. americanum (water extract) & 9.47 & 2.22 & 4.66 & 13.45 & - \\
\hline Infected control & 3.53 & 0.26 & 4.91 & - & - \\
\hline Naïve & 3.81 & - & 2.72 & 2.46 & - \\
\hline
\end{tabular}

\section{References:-}

1. Bourke C, Nausch N, Rujeni N, Appleby LJ, Mitchell K, Midzi N, Mduluza T, Mutapi F. (2013) Integrated Analysis of Innate, Th1, Th2, Th17, and Regulatory Cytokines Identifies Changes in Immune Polarisation Following Treatment of Human Schistosomiasis. J. Infect. Dis. 208(1):159-169

2. Carson FL (2007) Histotechnology 2nd ed, Chicago: ASCP Press

3. Carson FL, Christa H (2009) Histotechnology: A Self-Instructional Text (3 ed.). Hong Kong: American Society for Clinical Pathology Press. pp 2.

4. Cheever AW (1992) Pathogenesis of Schistosoma mansoni infection. Mem.Inst.Oswaldo Cruz 87(4):337-340.

5. Cheever AW, Jankovic D, Yap GS, Kullberg MC, Sher A, Wynn T.A. (1998) Role of cytokines in the formation and downregulation of hepatic circumoval granulomas and hepatic fibrosis in Schistosoma mansoniinfected mice, Mem Inst Oswaldo Cruz 93(1):25-32

6. Chiaramonte MG, Donaldson DD, Cheever AW, Wynn TA (1999) An IL-13 inhibitor blocks the development of hepatic fibrosis during a T-helper type 2-dominated inflammatory response. J.Clin.Invest 104(6):777-785.

7. Chiaramonte MG, Schopf LR, Neben TY, Cheever AW, Donaldson DD, \& Wynn TA. (1999) IL-13 is a key regulatory cytokine for Th2 cell-mediated pulmonary granuloma formation and IgE responses induced by Schistosoma mansoni eggs. J.Immunol 162(2):920-930.

8. Chitsulo L, Engels D, Montresor A, Savioli, L. (2000) The global status of schistosomiasis and its control. Acta Trop 77(1):41-51.

9. Cintron-Rivera AA, Diaz-Rivera RS, Garcia-Palmieri MR, Gonzalez O, Koppisch E, Marchand E.J, RamosMorales F, Torregrosa MV (1956) Acute Manson's schistosomiasis. Am.J.Med 21(6):918-943.

10. Cioli D, Pica-Mattoccia L, Basso A, Guidi A (2014) Schistosomiasis control: praziquantel forever?. Mol. Biochem. Parasitol 195(1):23-29.

11. de Pasquale, A. (1984) Pharmacognosy: the oldest modern science. J.Ethnopharmacol 11(1):1-16.

12. Doenhoff M, Bickle Q, Long E, Bain J, McGregor A (1978) Factors affecting the acquisition of resistance against Schistosoma mansoni in the mouse. I. Demonstration of resistance to reinfection using a model system that involves perfusion of mice within three weeks of challenge. J. Helminthol 52:173- 186.

13. Fallon PG, Richardson EJ, McKenzie GJ, McKenzie AN (2000) Schistosome infection of transgenic mice defines distinct and contrasting pathogenic roles for IL-4 and IL-13: IL-13 is a profibrotic agent. J.Immunol 164(5):2585-2591.

14. Farah IO, Nyindo M, King CI, Hau J (2000) Hepatic granulomatous response to Schistosoma mansoni eggs in BALB/C mice and Olive baboons (Papiocynocephalus Anubis). J. Comp. Pathol 123:7-14.

15. Fenwick A, Savioli L, Engels D, Robert BN, Todd MH (2003) Drugs for the control of parasitic diseases: current status and development in schistosomiasis. Trends Parasitol 19(11):509-515.

16. Flisser A and McLaren DJ (1989) Effect of praziquantel treatment on lung-stage larvae of Schistosoma mansoni in vivo. Parasitol 98(02):203-211.

17. Frohberg H (1984) Results of toxicological studies on praziquantel. Arzneimittel-Forschung, 34(9B), pp.11371144

18. Gonnert R and Andrews P (1977) Praziquantel, a new board-spectrum antischistosomal agent. Z Parasitenkd 52:129-150. 
19. Hopwood D (1996) Fixation and fixatives. In: Bancroft J, Stevens A (ed) Theory and practice of histological techniques. New York: Churchill Livingstone

20. Ismail M, Botros S, Metwally A, William S, Farghally A, Tao LF, Day TA and Bennett JL (1999) Resistance to Praziquantel: direct evidence from Schistosoma mansoni isolated from Egyptian villagers. Am.J.Trop.Med.Hyg 60(6):932-935.

21. Kabatereine NB, Kemijumbi J, Ouma JH, Sturrock RF, Butterworth AE, Madsen H, Ørnbjerg N, Dunne DW, Vennnervald BJ (2003) Efficacy and side effects of praziquantel treatment in a highly endemic Schistosoma mansoni focus at Lake Albert, Uganda. Trans R Soc Trop Med Hyg, 97(5):599-603.

22. Katz N, Rocha RS, Chaves A, (1979) Preliminary trials with Praziquantel in human infections due to Schistosoma mansoni. Bull World Health Organ 57:781-785.

23. McManus DP, Loukas A (2008) Current status of vaccines for schistosomiasis. Clin. Microbiol. Rev 21(1):225242

24. Mitchell K, Mutapi F, Savill N, Woolhouse M (2012) Protective immunity to Schistosoma haematobium infection is primarily an anti-fecundity response stimulated by the death of adult worms. Proc Natl Acad Sci U S A 109:13347-13352

25. Moilo JM, Mkoji GM, Keriko JM, Yole DS (2014) Anti-Schistosomal activity of Chenopodium ambrosoides extracts in adult worms in vivo and in vitro. Journal of Natural Sciences Research 4:12

26. Muchirah PN, Yole D, Kutima H, Waihenya R, Kuria KM, John M (2012) Determination of effective praziquantel dose in different mouse strains: BALB/c and Swiss mice in treatment of Schistosoma mansoni. J. Clin. Immunol. Immunopathol. Res., 4(2), pp.12-21.

27. Mutapi F, Burchmore R, Mduluza T, Foucher A, Harcus Y, Nicoll G, Midzi N, Turner CM, Maizels RM (2005) Praziquantel treatment of individuals exposed to Schistosoma haematobium enhances serological recognition of defined parasite antigens. J Infect Dis 192:1108-1118.

28. Nash TE, Cheever AW, Ottesen EA, Cook JA (1982) Schistosome infections in humans: perspectives and recent findings. NIH conference, Ann.Intern.Med, 97(5)740-754.

29. Nwaka S, Ridley R, (2003) Virtual drug discovery and development for neglected diseases through public private partnerships. Nat. Rev. Drug Discov 2:919-928

30. Osebe T, Mbaria J, Yole D, Odongo D, Nderitu J, Ochanda H (2016) Bioactivity and toxicity of Bridelia micrantha, Chenopodium ambrosoides and Ocimum americanum plant extracts. Int J Basic Clin Pharmacol 6(1):5-11.

31. Ramadan ME, Ramadan MEE, Yousef MSM (2013) Role of TNF alpha in schistosoma mansoni infection and cirrhotic liver, International Journal of Pharmaceutical and Medical Research, 1(1):6-12

32. Rates SMK (2001) Plants as source of drugs. Toxicon 39:603-613

33. Sabah AA, Fletcher C, Webbe G, Doenhoff MJ (1986) Schistosoma mansoni: chemotherapy of infections of different ages. Exp Parasitol 61: 294-303

34. Sepulveda J, Tremblay JM, DeGnore JP, Skelly PJ, Shoemaker CB (2010) Schistosoma mansoni host-exposed surface antigens characterized by sera and recombinant antibodies from schistosomiasis-resistant rats. Int $\mathbf{J}$ Parasitol, 40(12): 1407-1417

35. Smithers SR, Terry RJ (1965) The infection of laboratory hosts with cercariae of Schistosoma mansoni and the recovery of the adult worms. Parasitol 55(4):695-700.

36. Tweyongyere R, Mawa PA, Kihembo M, Jones FM, Webb EL, Cose S, Dunne DW, Vennervald BJ, Elliott AM (2011) Effect of praziquantel treatment of Schistosoma mansoni during pregnancy on immune responses to schistosome antigens among the offspring: results of a randomised, placebo-controlled trial. BMC Infect Dis 11:234

37. van der Werf MJ, De Vlas SJ, Brooker S, Looman CW, Nagelkerke NJ, Habbema JD, Engels D (2003) Quantification of clinical morbidity associated with schistosome infection in sub-Saharan Africa. Acta Trop 86(2-3):125-139.

38. Waiganjo N, Yole D, Ochanda H (2014) Anti-Schistosomal activity of five plant extracts on Swiss white mice infected with Schistosoma mansoni. IOSR J Pharm Biol Sci 9(1):49-53

39. Warren KS (1973) The pathology of schistosome infections. Helminth. Abstr. 42:592-633

40. WHO (2002) Prevention and Control of Schistosomiasis and Soil-Transmitted Helminthiasis: Report of a WHO Expert Committee. WHO Technical Report Series No. 912 Geneva, Switzerland: World Health Organization. 